\title{
Treatment patterns, resource utilization, and outcomes among hospitalized patients with methicillin-resistant Staphylococcus aureus complicated skin and soft tissue infections in Lebanon and Saudi Arabia
}

This article was published in the following Dove Press journal:

Infection and Drug Resistance

3 February 2017

Number of times this article has been viewed

\author{
Madonna J Matar' \\ Rima Moghnieh ${ }^{2}$ \\ Adel F Alothman ${ }^{3}$ \\ Abdulhakeem $\bigcirc$ Althaqafi ${ }^{4}$ \\ Thamer $\mathrm{H}$ Alenazi ${ }^{3}$ \\ Fayssal M Farahat ${ }^{4}$ \\ Shelby Corman ${ }^{5}$ \\ Caitlyn T Solem ${ }^{5}$ \\ Nirvana Raghubir ${ }^{6}$ \\ Cynthia Macahilig ${ }^{7}$ \\ Seema Haider ${ }^{8}$ \\ Jennifer M Stephens ${ }^{5}$
}

'Department of Infectious Diseases, Notre Dame des Secours University Hospital, Jbeil, Lebanon; ' 2 Department of Internal Medicine, Makassed Genera Hospital, Beirut, Lebanon; ${ }^{3}$ College of Medicine, King Saud bin Abdulaziz University for Health Sciences, King Abdullah International Medical Research Center, Riyadh, Saudi Arabia; ${ }^{4}$ King Abdullah International Medical Research Center, Infection Prevention and Control, King AbdulAziz Medical City, King Saud bin AbdulAziz University for Health Sciences, Jeddah, Saudi Arabia; ${ }^{5}$ Pharmerit International, Real-World Evidence/Data Analytics, Bethesda, MD, ${ }^{6}$ Pfizer, New York, NY, ${ }^{7}$ Medical Data Analytics, Parsippany, NJ, ${ }^{8}$ Pfizer, Groton, CT, USA

Correspondence: Jennifer M Stephens Pharmerit International, 4350 East West Highway, Suite 430, Bethesda, MD 208I4, USA

$\mathrm{Tel}+\mathrm{I} 30|82| 1290$

$\mathrm{Fax}+\mathrm{I} 3018211296$

Email jstephens@pharmerit.com
Objectives: To describe treatment patterns and medical resource use for methicillin-resistant Staphylococcus aureus (MRSA) complicated skin and soft tissue infections (cSSTI) in Saudi Arabia and Lebanon in terms of drug selection against the infecting pathogen as well as hospital resource utilization and clinical outcomes among patients with these infections.

Methods: This retrospective chart review study evaluated 2011-2012 data from five hospitals in Saudi Arabia and Lebanon. Patients were included if they had been discharged with a diagnosis of MRSA cSSTI, which was culture-proven or suspected based on clinical criteria. Hospital data were abstracted for a random sample of patients with each infection type to capture demographics, treatment patterns, hospital resource utilization, and clinical outcomes Statistical analysis was descriptive.

Results: Data were abstracted from medical records of 87 patients with MRSA cSSTI; mean age $52.4 \pm 25.9$ years and $61 \%$ male. Only $64 \%$ of patients received an MRSA active initial therapy, with $56 \%$ of first-line regimens containing older beta-lactams. The mean total length of stay was 26.3 days, with the majority (19.1 days) spent in general wards. Surgical procedures included incision and drainage (22\% of patients), debridement (14\%), and amputation (5\%). Mechanical ventilation was required by $9 \%$ of patients, with a mean duration of 18 days per patient. Hemodialysis was required by four patients $(5 \%)$, two of whom were reported to have moderate to severe renal disease on admission, for a mean of 5.5 days. Inpatient mortality was $8 \%$. Thirty-nine percent were prescribed at least one antibiotic at discharge, with the most commonly prescribed discharge antibiotics being clindamycin (44\%), ciprofloxacin (18\%), trimethoprim/sulfamethoxazole (12\%), and linezolid (9\%).

Conclusion: This Middle Eastern real-world study of resource use and treatment patterns in MRSA cSSTI indicates that management of this condition could be further optimized in terms of drug selection and resource utilization.

Keywords: antibiotics, length of stay, Saudi Arabia, Lebanon, skin, skin structure infections

\section{Introduction}

Staphylococcus aureus is a gram-positive bacterium frequently implicated in a variety of infections, including complicated skin and soft tissue infections (cSSTI). ${ }^{1}$ S. aureus is well recognized as a major cause of nosocomial infections ${ }^{1}$ and is associated with high morbidity and mortality with rapid development of antibiotic resistance. ${ }^{2}$ Overall, in 
developed countries, methicillin-resistant S. aureus (MRSA) accounts for $22 \%$ of gram-positive cocci isolated from SSTIs but may be above $50 \%$ in some regions. ${ }^{1}$

In the developing world, surgical site infections (SSIs), a type of cSSTI, are the most common hospital-acquired infection; an SSI was reported in $5.6 \%$ of patients following a surgical procedure in developing countries. ${ }^{3}$ In the same report, the World Health Organization estimates that up to $33 \%$ of operated patients in countries with limited resources develop an SSI. S. aureus is a common cause of SSIs in both low- and high-income countries. Specifically in low-income countries, S. aureus was identified as the causative pathogen in $\sim 20 \%$ of SSIs, where $54 \%$ of these isolates were resistant to methicillin. ${ }^{3}$ Another study in Saudi Arabia found that while 39.5\% of $S$. aureus isolates were MRSA, $87 \%$ of these MRSA isolates were associated with wound, skin, and soft tissues infections. ${ }^{4}$

Despite the prevalence of MRSA in cSSTI, data on realworld treatment patterns and resource utilization burden in the Middle East are limited. The objectives of this study were to describe the treatment received by patients in Saudi Arabia and Lebanon in terms of drug selection against the infecting pathogen, and document hospital resource utilization and clinical outcomes among patients with these infections.

\section{Methods}

\section{Study design}

This study is a retrospective, observational medical chart review that took place at a total of five study sites in Saudi Arabia (two sites) and Lebanon (three sites). Per the study protocol, the SI assessed the need for ethics/IRB application in their site and IRB waiver or approval was completed at all sites based on local requirements. Specifically, approval was obtained from Kingdom of Saudi Arabia, National Guard Health Affairs, King Abdalaziz Medical City- Jeddah and Riyadh; Ain Wazein Hospital IRB; and Makassed General Hospital, Riad El-Solh Beirut, Lebanon. In all cases, written informed consent was not required as this is a non-interventional medical chart review study.

\section{Inclusion and exclusion criteria}

Hospital admissions occurring between January 1, 2011 and December 31, 2012 were initially screened for inclusion using electronic medical records to identify patients who had been discharged with a diagnosis of MRSA cSSTI during the study years. In order to be included for chart abstraction, patients had to have suspected or culture-proven MRSA cSSTI with appropriate dermatological manifestations. ${ }^{5}$ For suspected cases without culture proof, patients needed to have evidence of dermatological manifestations and at least two of the following symptoms for inclusion: fever (body temperature $\geq 38.5^{\circ} \mathrm{C}$ ); hypothermia (body temperature $\leq 35.5^{\circ} \mathrm{C}$ ); respiratory rate $>30$ breaths per minute; systolic hypotension $(<90$ $\mathrm{mmHg}$ ); heart rate $>120$ beats per minute; elevated peripheral white blood cell count $>10,000 / \mathrm{mm}^{3}$; leukopenia with total white blood cell count $<4,500$ cells $/ \mathrm{mm}^{3}$; and/or elevated inflammatory markers (eg, procalcitonin or C-reactive protein). Patients were excluded if they had inadequate data on the treatments and outcomes of interest or hospital resource utilization for the study, as judged by the investigators.

\section{Data collection}

Data were collected from patient medical records by hospitalbased infectious disease specialists, medical microbiologists, internal medicine specialists, surgeons, and intensivists. All data collection instruments were translated into local language and, prior to roll-out, pilot-tested to ensure relevance and clarity of data points to be collected.

Extracted chart data included demographics and comorbid conditions; antibiotic treatment patterns, including initial and subsequent inpatient antibiotic regimens prescribed and antibiotic therapy prescribed at discharge; health care resource utilization, including surgeries, other procedures, laboratory tests, and length of stay (LOS) in hospital; and clinical outcomes, including clinical response at discharge and rehospitalization.

Antibiotics received during hospitalization were categorized as MRSA-active and not MRSA-active. MRSA-active antibiotics as defined by guidelines included vancomycin (intravenous only), teicoplanin (intravenous only), clindamycin, doxycycline, trimethoprim/sulfamethoxazole, linezolid, fusidic acid, rifampin, and tigecycline. ${ }^{6}$ Antibiotics categorized as not MRSA-active included beta-lactams (during the timeframe of data collection, ceftobiprole and ceftaroline were not available) and gentamicin. In the absence of institution-specific antibiograms, fluoroquinolones and carbapenems were only categorized as MRSA-active if culture results indicated sensitivity to the respective antibiotic.

\section{Statistical analysis}

Unless otherwise listed, binary and categorical endpoints were summarized in terms of percentages in each category and continuous variables were presented using number of observations (when less than the entire sample), arithmetic mean, and standard deviation.

\section{Results}

\section{Demographic and clinical characteristics}

Detailed medical chart data were collected for 87 patients with MRSA cSSTI across the five study sites, 49 from 
Table I Patient profile: MRSA cSSTI

\begin{tabular}{|c|c|c|c|}
\hline & $\begin{array}{l}\text { Overall } \\
(N=87)\end{array}$ & $\begin{array}{l}\text { Saudi Arabia } \\
(\mathrm{N}=49)\end{array}$ & $\begin{array}{l}\text { Lebanon } \\
(\mathrm{N}=38)\end{array}$ \\
\hline Male & $61 \%$ & $61 \%$ & $61 \%$ \\
\hline Age at hospital admission, mean \pm SD & $52.4 \pm 25.9$ & $52.1 \pm 27.6$ & $52.8 \pm 23.9$ \\
\hline \multicolumn{4}{|l|}{ Weight at hospital admission (kg) } \\
\hline $\mathrm{N}$ with information available & 66 & 34 & 32 \\
\hline Mean \pm SD & $70.5 \pm 28.6$ & $69.7 \pm 25.1$ & $71.3 \pm 32.2$ \\
\hline \multicolumn{4}{|l|}{ Body Mass Index (mg/m²) } \\
\hline $\mathrm{N}$ with information available & 52 & 30 & 22 \\
\hline Mean \pm SD & $27.2 \pm 8.7$ & $27.3 \pm 7.8$ & $27.2 \pm 10.0$ \\
\hline Number of reported comorbidities, mean \pm SD & $2.2 \pm 1.9$ & $1.9 \pm 1.9$ & $2.4 \pm 1.9$ \\
\hline Diabetes & $49 \%$ & $49 \%$ & $50 \%$ \\
\hline Coronary artery disease & $26 \%$ & $14 \%$ & $42 \%$ \\
\hline Peripheral vascular disease & $20 \%$ & $18 \%$ & $21 \%$ \\
\hline Moderate to severe renal disease & $20 \%$ & $22 \%$ & $16 \%$ \\
\hline Congestive heart failure & $16 \%$ & $10 \%$ & $24 \%$ \\
\hline Cerebrovascular disease & $11 \%$ & $10 \%$ & $13 \%$ \\
\hline
\end{tabular}

Abbreviations: cSSTI, complicated skin and soft tissue infections; MRSA, methicillin-resistant Staphylococcus aureus; SD, standard deviation.

Saudi Arabia, and 38 from Lebanon. The majority of admissions were for medical reasons $(86 \%)$, with the remaining attributed to surgical/trauma (14\%). The mean number of comorbid conditions per patient was 2.2 , with the most common conditions being diabetes, coronary artery disease, and peripheral vascular disease (Table 1).

Of the 84 patients who had at least one culture collected, $81(96 \%)$ had at least one positive result. Positive cultures were most commonly collected from wounds $(83 \%$ of patients) and blood (17\%). Susceptibility testing for at least one antibiotic was performed in 80 isolates. Figure 1 shows susceptibility results for these isolates. All tested isolates were sensitive to vancomycin $(n=78$ tested) and teicoplanin
( $n=34$ tested). Only five isolates were tested for linezolid sensitivity, and all were found to be sensitive.

\section{Antibiotic treatment patterns}

The 87 MRSA cSSTI patients received a total of 131 unique antibiotics designated by study investigators to be first-line therapy (as some regimens consisted of multiple antibiotics), with only $64.4 \%$ receiving an MRSA-active treatment as part of their first line. Fifty-six percent of patients received beta-lactams (thus representing inactive therapy), and the most frequently prescribed MRSA-active antibiotic was vancomycin (38\%) in the initial treatment line (Table 2). Among the 33 patients (38\%) requiring a total

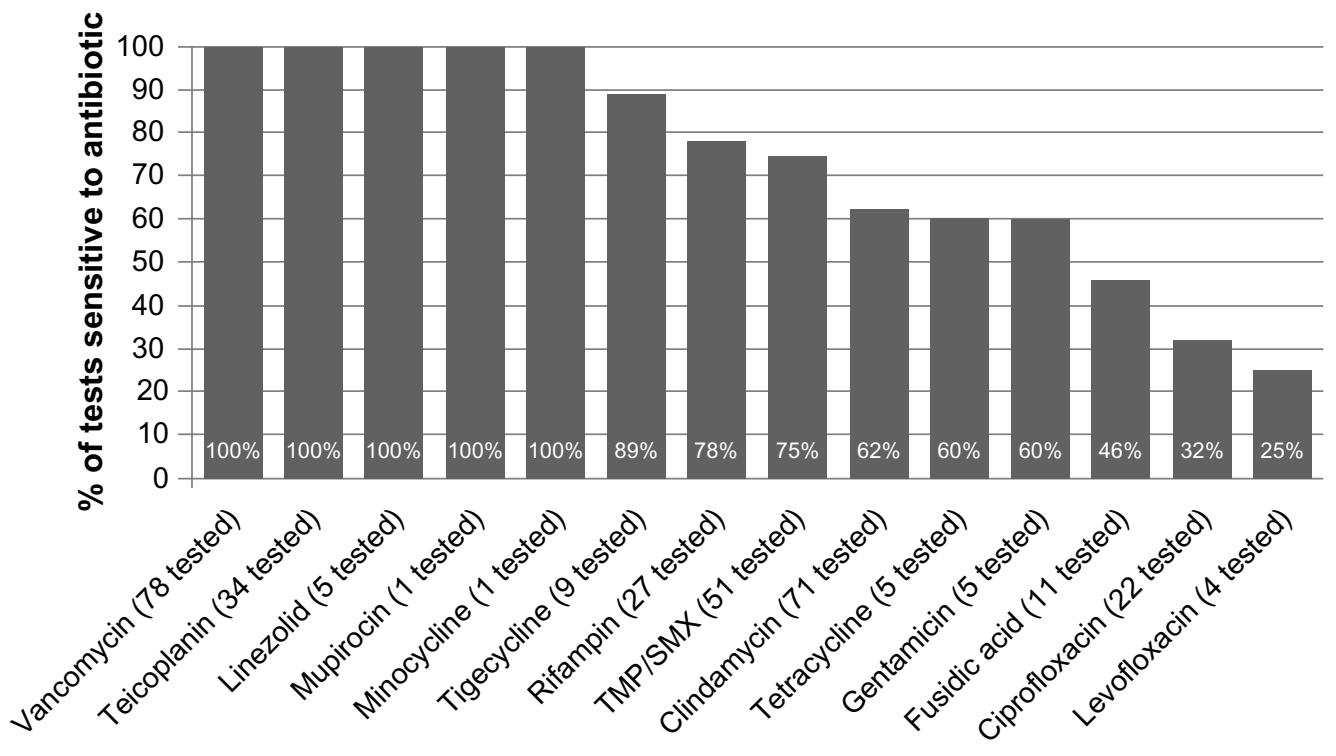

Figure I MRSA cSSTI susceptibility profile.

Abbreviations: cSSTI, complicated skin and soft tissue infections; MRSA, methicillin-resistant Staphylococcus aureus; TMP/SMX, trimethoprim/sulfamethoxazole. 
Table 2 Antibiotics prescribed by line of treatment and country

\begin{tabular}{|c|c|c|c|}
\hline & $\begin{array}{l}\text { Total patients } \\
(\mathbf{N}=87)\end{array}$ & $\begin{array}{l}\text { Saudi Arabia } \\
(N=49)\end{array}$ & $\begin{array}{l}\text { Lebanon } \\
(\mathrm{N}=38)\end{array}$ \\
\hline Initial treatment, $\mathrm{N}(\%)$ & $100 \%$ & $100 \%$ & $100 \%$ \\
\hline Beta-lactam antibiotic ${ }^{a}$ & $56 \%$ & $51 \%$ & $63 \%$ \\
\hline Vancomycin ${ }^{\mathrm{a}}$ & $38 \%$ & $53 \%$ & $18 \%$ \\
\hline Clindamycin ${ }^{\mathrm{a}}$ & $18 \%$ & $31 \%$ & $3 \%$ \\
\hline Teicoplanin ${ }^{\mathrm{a}}$ & $10 \%$ & $0 \%$ & $24 \%$ \\
\hline Meropenem $^{\mathrm{a}}$ & $7 \%$ & $10 \%$ & $3 \%$ \\
\hline Ciprofloxacin ${ }^{\mathrm{a}}$ & $7 \%$ & $8 \%$ & $5 \%$ \\
\hline Ceftriaxone ${ }^{a}$ & $5 \%$ & $8 \%$ & $0 \%$ \\
\hline Cefazolin $^{\mathrm{a}}$ & $5 \%$ & $8 \%$ & $0 \%$ \\
\hline Gentamicin $^{\mathrm{a}}$ & $5 \%$ & $8 \%$ & $0 \%$ \\
\hline Erythromycin ${ }^{\mathrm{a}}$ & $3 \%$ & $0 \%$ & $8 \%$ \\
\hline Second-line treatment & $38 \%$ & $33 \%$ & $45 \%$ \\
\hline Vancomycin ${ }^{\mathrm{a}}$ & $33 \%$ & $50 \%$ & $18 \%$ \\
\hline Teicoplanin ${ }^{\mathrm{a}}$ & $27 \%$ & $0 \%$ & $53 \%$ \\
\hline Clindamycin $^{\mathrm{a}}$ & $21 \%$ & $31 \%$ & $12 \%$ \\
\hline Meropenem $^{\mathrm{a}}$ & $6 \%$ & $6 \%$ & $6 \%$ \\
\hline Ciprofloxacin ${ }^{\mathrm{a}}$ & $6 \%$ & $6 \%$ & $6 \%$ \\
\hline $\begin{array}{l}\text { Trimethoprin/ } \\
\text { sulfamethoxazole }^{\text {a }}\end{array}$ & $6 \%$ & $13 \%$ & $0 \%$ \\
\hline Linezolid $^{\mathrm{a}}$ & $3 \%$ & $6 \%$ & $0 \%$ \\
\hline Tigecycline $^{\mathrm{a}}$ & $3 \%$ & $0 \%$ & $6 \%$ \\
\hline Third-line treatment & $5 \%$ & $8 \%$ & $0 \%$ \\
\hline Linezolid ${ }^{\mathrm{a}}$ & $50 \%$ & $50 \%$ & - \\
\hline Beta-lactam antibiotic ${ }^{a}$ & $25 \%$ & $25 \%$ & - \\
\hline Rifampin $^{\mathrm{a}}$ & $25 \%$ & $25 \%$ & - \\
\hline Vancomycin ${ }^{\mathrm{a}}$ & $25 \%$ & $25 \%$ & - \\
\hline Gentamicin $^{\mathrm{a}}$ & $25 \%$ & $25 \%$ & - \\
\hline
\end{tabular}

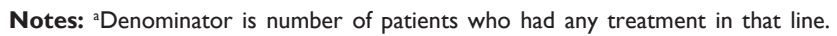
Drug categories are not mutually exclusive (ie, patients could use more than one antibiotic in each line). of 36 second-line antibiotics, vancomycin remained the most commonly prescribed antibiotic (to $33 \%$ of patients), followed by teicoplanin (27\%) and clindamycin (21\%). Linezolid was received by two of the four patients who received third-line treatment, with the others receiving a beta-lactam $(\mathrm{n}=1)$ and vancomycin $(\mathrm{n}=1)$. Overall, 75 patients $(86 \%)$ received at least one antibiotic considered to be MRSA-active.

A total of 34 patients $(39 \%)$ were prescribed at least one antibiotic regimen at discharge. The most commonly prescribed discharge antibiotics were clindamycin (44\%), ciprofloxacin (18\%), trimethoprim/sulfamethoxazole (12\%), and linezolid (9\%).

\section{Health care resource utilization}

Overall, the mean total LOS was $26.3 \pm 34.1$ days, with the majority (19.1 days) spent in general wards (Figure 2). LOS was higher in Saudi Arabia (35.5 \pm 40.8 days) than in Lebanon (14.5 \pm 16.8 days), with fewer days spent in high-dependency/ intermediate units. The most common surgical procedures performed in patients with MRSA cSSTI included incision and drainage (22\% of patients) and debridement (14\%). Mechanical ventilation was required by $9 \%$ of patients, with a mean duration of 18 days per patient. Hemodialysis was required by four patients (5\%) for a mean of 5.5 days. Two of these patients were reported to have moderate to severe renal

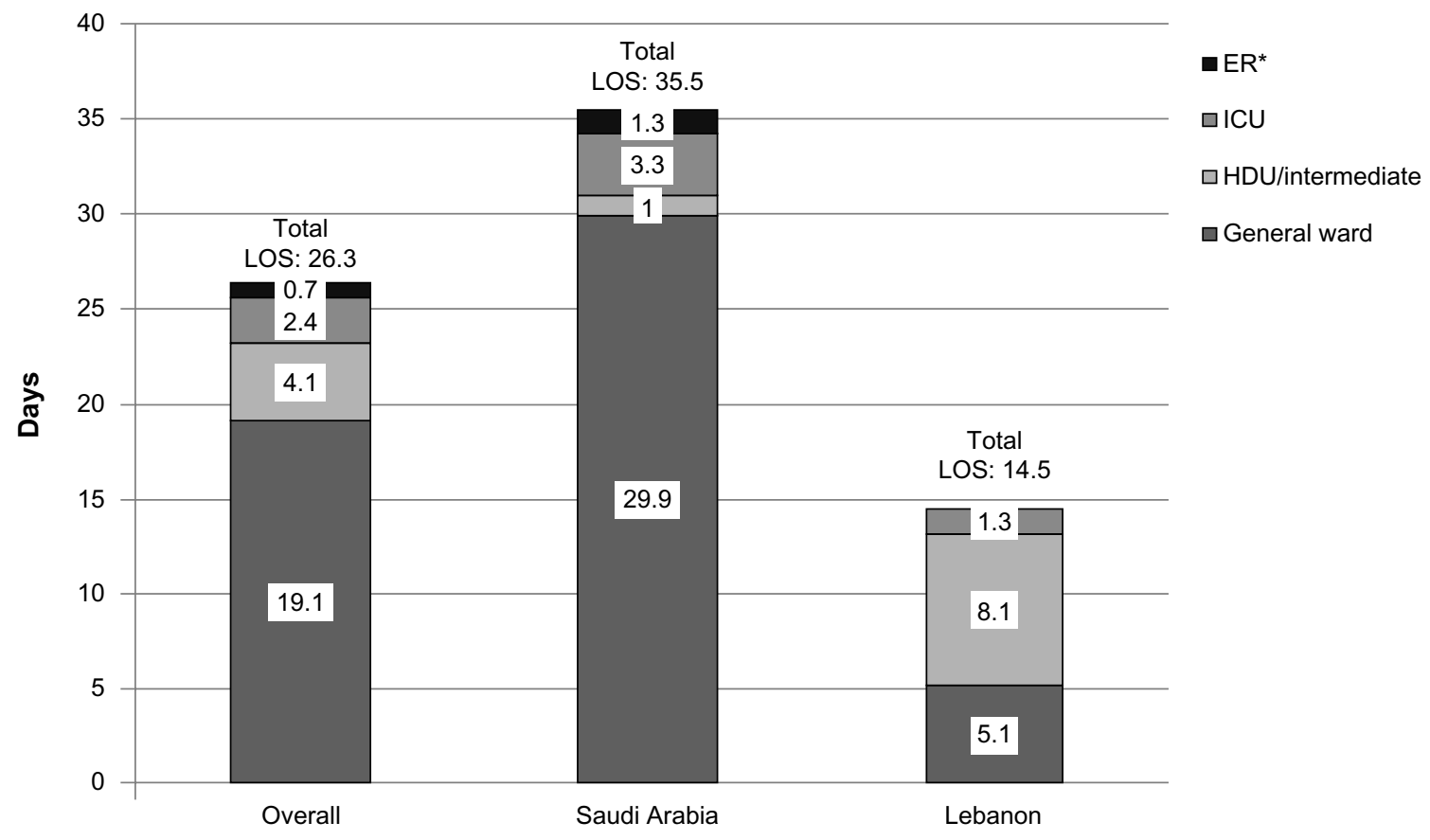

Figure 2 Mean length of hospital stay in patients with MRSA cSSTI.

Note: *In Saudi Arabia, patients may stay in the emergency room (ER) for several days before being transferred to another ward.

Abbreviations: cSSTI, complicated skin and soft tissue infections; HDU, high dependency unit; ICU, intensive care unit; LOS, length of stay; MRSA, methicillin-resistant Staphylococcus aureus. 
Table 3 MRSA cSSTI clinical outcomes

\begin{tabular}{|c|c|c|c|}
\hline Outcome & $\begin{array}{l}\text { Overall } \\
(\mathrm{N}=87)\end{array}$ & $\begin{array}{l}\text { Saudi Arabia } \\
(\mathrm{N}=49)\end{array}$ & $\begin{array}{l}\text { Lebanon } \\
(\mathbf{N}=\mathbf{3 8})\end{array}$ \\
\hline \multicolumn{4}{|l|}{ Clinical response at discharge } \\
\hline Cure $^{a}$ & $44 \%$ & $47 \%$ & $39 \%$ \\
\hline Improvement $^{\mathrm{b}}$ & $33 \%$ & $20 \%$ & $50 \%$ \\
\hline Failure $^{c}$ & $7 \%$ & $8 \%$ & $5 \%$ \\
\hline Indeterminate ${ }^{d}$ & $16 \%$ & $24 \%$ & $5 \%$ \\
\hline \multicolumn{4}{|l|}{ Outcomes 30 -days postdischarge ${ }^{e}$} \\
\hline Relapse of MRSA cSSTI & $6 \%$ & $7 \%$ & $5 \%$ \\
\hline Rehospitalization for MRSA cSSTI & $4 \%$ & $5 \%$ & $3 \%$ \\
\hline Rehospitalization, any reason & $14 \%$ & $7 \%$ & $22 \%$ \\
\hline
\end{tabular}

Note: aResolution of all signs and symptoms/improvement to such an extent that further antimicrobial therapy was not necessary. ${ }^{\circ}$ Improvement in signs and symptoms. 'Persistence, incomplete clinical resolution, or worsening in signs and symptoms. Inability to determine an outcome. ePostdischarge outcomes only measured in patients who survived to discharge (80 overall, 43 Saudi Arabia, 37 Lebanon).

Abbreviations: cSSTI, complicated skin and soft tissue infections; MRSA, methicillin-resistant Staphylococcus aureus.

disease on admission, but whether patients were receiving dialysis prior to admission is not known.

\section{Clinical outcomes}

Clinical outcomes among patients with MRSA cSSTI at discharge and 30 days postdischarge are shown in Table 3. Over three-quarters of patients achieved cure or improvement by the time of discharge, and inhospital mortality was $8 \%$. Of the patients who survived to discharge $(\mathrm{n}=80)$, few patients $(6 \%)$ had a relapse of cSSTI, with even fewer $(4 \%)$ hospitalized for relapse.

\section{Discussion}

To our knowledge, this is the first medical chart review study that provides a snapshot of current drug treatment patterns and resource use for MRSA cSSTI in Saudi Arabia and Lebanon. We found that beta-lactams were a frequent initial antibiotic choice $(56 \%)$ despite being inactive for MRSA, while vancomycin was prescribed only $38 \%$ and teicoplanin $10 \%$ of the time as first-line treatment. ${ }^{7}$

Previous studies in this region have only focused on laboratory isolates and resistance patterns. Our study was consistent with previous reports in Saudi Arabia indicating 100\% sensitivity of MRSA to vancomycin, teicoplanin, and linezolid; ${ }^{4}$ however, these MRSA-specific therapies appeared to be reserved for second- and third-line treatments in our study. It is unclear whether economic barriers were driving the delayed MRSAactive treatment seen in our observational study.

Prescribing inadequate coverage for the initial treatment of cSSTI has been reported to worsen outcomes. A large hospital database study reported that failure at initial antibiotic therapy was associated with around three times increased mortality (mortality odds ratio 2.91, 95\% confidence interval, 2.34, 3.62; $P<0.1)$ and increased hospital LOS by $\sim 5$ days. ${ }^{8}$

Diabetes was a frequent comorbidity $(\sim 50 \%)$ in this Middle East population with MRSA cSSTI. Evidence suggests that the rate of cellulitis is more than two times higher in patients with diabetes than in patients without diabetes (incidence rate ratio, $2.45 ; P<0.001) .{ }^{9}$ In patients with diabetes and foot infections, the estimated prevalence of MRSA ranged from a low of $15 \%$ to a high of $50 \%{ }^{10}$ Given the added risk in patients with diabetes, our study suggests that consideration should be given earlier to MRSA etiology when the patient is admitted to the hospital for treatment of cSSTI.

A number of study limitations are common with retrospective data collection; nevertheless, this initial attempt at describing detailed treatment and resource use information may help inform future studies aimed at testing new strategies to enhance care and reduce burden of MRSA cSSTI in the Middle East. One example of this limitation is that it was not possible to determine whether ventilation was required due to an MRSA complication.

\section{Conclusion}

This Middle Eastern real-world study examined the resource use and treatment patterns of MRSA cSSTI in Saudi Arabia and Lebanon. Patients were frequently prescribed MRSA-inactive initial lines of treatment and had lengths of stay between 14 to 36 days. Inhospital mortality was $8 \%$; however, over half of patients were discharged with cure or improvement. Our findings indicate that the management of MRSA cSSTI could be further optimized in the Middle East. The delay to start MRSA active treatment should be further examined in the region to include LOS impact of suboptimal therapy.

\section{Acknowledgments}

The authors would like to thank Monica Katyal for her assistance with data collection and analysis. This study was sponsored by Pfizer.

\section{Author contributions}

AFA, AOA, MJM, RM, NR, SH, CTS, and JMS contributed to study design. CM, AFA, AOA, MJM, RM, THA, and FF were involved in data acquisition. CTS and SC undertook data analysis. All authors contributed to data interpretation, manuscript drafting, and approved the final manuscript.

\section{Disclosure}

Shelby Corman, Jennifer Stephens, and Caitlyn Solem are employees of Pharmerit International who were paid 
consultants of Pfizer in connection with the development of this manuscript and study design, management, and statistical analysis for the study. Cynthia Macahilig is an employee of Medical Data Analytics, a subcontractor to Pharmerit International, who was a paid consultant of Pfizer in the study design, management, and data collection for the study. Nirvana Raghubir and Seema Haider are employees and shareholders of Pfizer. Adel Alothman received honoraria for several presentations from Pfizer, MSD, and Alhikma; received travel support from Pfizer, MSD, Gilead, and Alhikma to attend symposia; and received honoraria for patient data collection related to this study from Pfizer. Abdulhakeem Althaqafi received a research grant (RR13/248/J) sponsored by Pfizer, and received honoraria for patient data collection related to this study from Pfizer. Fayssal Farahat received honoraria for patient data collection related to this study from Pfizer. Madonna Matar has received travel support for attending meetings, and received honoraria for patient data collection related to this study from Pfizer. Rima Moghnieh and received honoraria for patient data collection related to this study from Pfizer and sponsorship for attending medical meetings from Pfizer and MSD. Thamer Alenazi has received travel support for attending medical meetings from MSD and Pfizer. The authors report no other conflicts of interest in this work.

\section{References}

1. Sader HS, Farrell DJ, Jones RN. Antimicrobial susceptibility of Grampositive cocci isolated from skin and skin-structure infections in European medical centres. Int J Antimicrob Agents. 2010;36(1):28-32.
2. Zetola N, Francis JS, Nuermberger EL, Bishai WR. Communityacquired meticillin-resistant Staphylococcus aureus: an emerging threat. Lancet Infect Dis. 2005;5(5):275-286.

3. Allegranzi B, Bagheri Nejad S, Combescure C, et al. Burden of endemic health-care-associated infection in developing countries: systematic review and meta-analysis. Lancet. 2011;377(9761):228-241.

4. El Amin NM, Faidah HS. Methicillin-resistant Staphylococcus aureus in the western region of Saudi Arabia: prevalence and antibiotic susceptibility pattern. Ann Saudi Med. 2012;32(5):513-516.

5. Corey GR, Stryjewski ME. New rules for clinical trials of patients with acute bacterial skin and skin-structure infections: do not let the perfect be the enemy of the good. Clin Infect Dis. 2011;52(Suppl 7): S469-S476.

6. Liu C, Bayer A, Cosgrove SE, et al; Infectious Diseases Society of America. Clinical practice guidelines by the Infectious Diseases Society of America for the treatment of methicillin-resistant Staphylococcus aureus infections in adults and children. Clin Infect Dis. 2011;52(3):e18-e55.

7. Peng Y, Ye X, Li Y, et al. Teicoplanin as an effective alternative to vancomycin for treatment of MRSA infection in Chinese population: a meta-analysis of randomized controlled trials. PLoS One. 2013;8(11):e79782.

8. Edelsberg J, Berger A, Weber DJ, Mallick R, Kuznik A, Oster G. Clinical and economic consequences of failure of initial antibiotic therapy for hospitalized patients with complicated skin and skin-structure infections. Infect Control Hosp Epidemiol. 2008;29(2):160-169.

9. Hamilton EJ, Martin N, Makepeace A, Sillars BA, Davis WA, Davis TM. Incidence and predictors of hospitalization for bacterial infection in community-based patients with type 2 diabetes: the Fremantle diabetes study. PLoS One. 2013;8(3):e60502.

10. Lipsky BA, Tabak YP, Johannes RS, Vo L, Hyde L, Weigelt JA. Skin and soft tissue infections in hospitalised patients with diabetes: culture isolates and risk factors associated with mortality, length of stay and cost. Diabetologia. 2010;53(5):914-923.
Infection and Drug Resistance

\section{Publish your work in this journal}

Infection and Drug Resistance is an international, peer-reviewed openaccess journal that focuses on the optimal treatment of infection (bacterial, fungal and viral) and the development and institution of preventive strategies to minimize the development and spread of resistance. The journal is specifically concerned with the epidemiology of antibiotic

\section{Dovepress}

resistance and the mechanisms of resistance development and diffusion in both hospitals and the community. The manuscript management system is completely online and includes a very quick and fair peerreview system, which is all easy to use. Visit http://www.dovepress.com/ testimonials.php to read real quotes from published authors. 